TRANSACTIONS OF THE

AMERICAN MATHEMATICAL SOCIETY

Volume 278, Number 2, August 1983

EMBEDDING $L^{1}$ IN $L^{1} / H^{1}$

BY

J. BOURGAIN

\begin{abstract}
It is proved that $L^{1}$ is isomorphic to a subspace of $L^{1} / H^{1}$. More precisely, there exists a diffuse $\sigma$-algebra $\subseteq$ on the circle such that the corresponding expectation $\mathbf{E}: H^{\infty} \rightarrow L^{\infty}(\mathbf{C})$ is onto. The method consists in studying certain martingales on the product $\Pi^{\mathbf{N}}$.
\end{abstract}

1. Introduction. Let us start by fixing some terminology. As usual, $\Pi$ will denote the circle equipped with its Haar measure $m, H_{0}^{1}$ is the subspace of those $f \in L^{1}(\Pi)$ for which $\hat{f}(n)=0$ for $n \leqslant 0$ and $q: L^{1} \rightarrow L^{1} / H_{0}^{1}$ is the quotient map.

We are interested in the question whether or not there exists a linear embedding of the Banach space $L^{1}$ in the space $L^{1} / H_{0}^{1}$. We briefly indicate some motivation for this problem. First, it was (and still remains) an open question if the three-spaceproperty holds for $L^{1}$-embedding, i.e. suppose $X$ a Banach space, $Y$ a subspace of $X$. Is it true that whenever $L^{1}$ embeds in $X$, it also has to embed in either $Y$ or $X / Y$ ?

The problem is also unsolved in the particular case $X=L^{1}$ and $Y$ isomorphic to a dual space. It is not hard to show that an embedding of $L^{1}$ in $X / Y$ is then equivalent to the existence of a subspace $S$ of $X, S$ isomorphic to $L^{1}$ so that the quotient map $X \rightarrow X / Y$ is an isomorphism when restricted to $S$.

In the special situation $X=L^{1}(\Pi)$ and $Y=H_{0}^{1}$, the answer was unknown for some time. There was hope that this may provide a counterexample in view of the following result, due to W. B. Johnson (see [9]).

Proposition 1. No complemented subspace of $L^{1} / H_{0}^{1}$ is isomorphic to $L^{1}$.

This is a consequence of the fact that any operator $T: L^{1} / H^{1} \rightarrow L^{1}$ maps weakly compact sets onto norm compact sets. Let us sketch the argument.

Consider the identity map $I: L^{\infty} / H^{\infty} \rightarrow L^{1} / H^{1}$. Then $(T I)^{*}: L^{\infty} \rightarrow H^{\infty} \rightarrow H^{1}$ is integral and therefore nuclear (since $H^{1}$ satisfies the Radon-Nikodym property). Consequently, also $T I$ is nuclear. Given now a weakly null sequence $\left(x_{n}\right)_{n=1,2, \ldots}$ in $L^{1} / H^{1}$, it follows from the lifting property (see [9] for instance) that $x_{n}=q\left(f_{n}\right)$ where $\left\{f_{n} ; n=1,2, \ldots\right\}$ is a relatively weakly compact set in $L^{1}(\Pi)$. Therefore, for each $\varepsilon>0$, a truncation argument provides a bounded sequence $\left(g_{n}\right)$ in $L^{\infty}$ such that $\left\|f_{n}-g_{n}\right\|_{1}<\varepsilon$ for each $n$. Thus

$$
\left\|T x_{n}-T I \tilde{g}_{n}\right\| \leqslant\|T\|\left\|x_{n}-I \tilde{g}_{n}\right\|<\varepsilon\|T\| .
$$

Received by the editors April 22, 1982 and, in revised form, July 20, 1982

1980 Mathematics Subject Classification. Primary 46E15, 42A99, 60G45.

(C)1983 American Mathematical Society 0002-9947/82/0000-0794/\$03.50 
Because $T I$ is nuclear, the set $\left\{T I\left(\tilde{g}_{n}\right) ; n=1,2, \ldots\right\}$ is compact for each $\varepsilon>0$. So we conclude that $\left\{T x_{n}\right\}$ is compact, as announced.

Using Proposition 1, the following is proved in [2].

Proposition 2. There is no almost isometric embedding of the complex $L^{1}$ space in $L^{1} / H^{1}$.

Thus $d\left(S, L^{1}\right)>\gamma>1$ for each subspace $S$ of $L^{1} / H_{0}^{1}$, where $d$ is the Banach-Mazur distance (see $[\mathbf{8}, 9]$ for definitions). This observation allows us to define a natural distortion of $L^{1}$, by taking

$$
\|f\|=\|f\|_{1}+\|q(f)\|, \quad f \in L^{1}(\Pi) .
$$

Say that an operator $T: X \rightarrow Y$ is a semiembedding provided $T$ is one-one and maps the closed unit ball of $X$ on a norm-closed subset of $Y$. It can be shown that a semiembedding $T: L^{1} \rightarrow L^{1}$ has to fix an $L^{1}$-copy (i.e. is an isomorphism when restricted to a subspace $S$ of $L^{1}, S$ isomorphic to $L^{1}$ ). On the other hand, (see [3]):

Proposition 3. The restriction of the quotient map $q: L^{1} \rightarrow L^{1} / H_{0}^{1}$ to the subspace $L_{\mathbf{R}}^{1}$ of real functions in $L^{1}(\Pi)$ is a semiembedding.

No example is known of a semiembedding of $L^{1}$ in a Banach space $X$ not containing $L^{1}$.

Our purpose is to prove the existence of a natural embedding of $L^{1}$ in $L^{1} / H_{0}^{1}$. There exists a diffuse $\sigma$-algebra $\subseteq$ on $\Pi$ so that the restriction of $q$ to the complex $L^{1}(\Im)$-space is an isomorphism. More precisely:

THEOREM. There exists an increasing sequence $\left(n_{k}\right)$ of positive integers, such that if $\subseteq$ is the $\sigma$-algebra on $\Pi$ generated by the functions $\sigma_{k}(\theta)=\operatorname{sign} \cos n_{k} \theta$, then the restriction of $q$ to $L^{1}(\Im)$ is an isomorphism. Consequently, for this $\sigma$-algebra $\subseteq$, the expectation operator $\mathbf{E}: H^{\infty} \rightarrow L^{\infty}(\Im)$ is onto.

The argument presented here is rather delicate. In order to give the reader an idea how it is organised, we briefly outline the proof. We have to introduce the $\sigma$-algebra $\Im$ such that the inequality

$$
\left\|h-\mathbf{E}_{\odot}[h]\right\|_{1} \geqslant \delta\|h\|_{1}
$$

holds for each $h \in H_{0}^{1}$. But choosing the sequence $\left(n_{k}\right)$ sufficiently lacunary, it is enough to verify $(*)$ for functions $h$ with spectrum contained in a set of the form

$$
E=\left\{\Sigma^{\prime} \nu_{k} n_{k} ;\left|\nu_{k}\right| \leqslant a_{k} \text { for each } k\right\}
$$

where $\left(a_{k}\right)$ is a sequence of positive integers and $\left(n_{k}\right),\left(a_{k}\right)$ satisfy the transference property. Thus the $n_{k}$-frequencies can be replaced by independent variables. The space $H_{0}^{1} \cap L_{E}^{1}$ identifies with a subspace of the space $\mathcal{K} \subset L^{1}\left(\Pi^{\mathbf{N}}\right)$ of those functions $h=\sum h_{k}$ on $\Pi^{\mathbf{N}}$ such that each increment $h_{k}=h_{k}\left(x_{1}, \ldots, x_{k}\right)$ is an $H_{0}^{1}$-function in $x_{k}$. The required inequality now becomes

$$
\left\|h-\mathbf{E}_{\mathscr{F}}[h]\right\|_{1} \geqslant\|h\|_{1}
$$

for $h \in \mathcal{H}$, where $\mathscr{F}$ is a natural diadic product $\sigma$-algebra on $\Pi^{\mathbf{N}}$ (generated by the functions $\left.\sigma_{k}(x)=\operatorname{sign} \cos x_{k}\right)$. 
This reduction of the problem is worked out in $\S 4$. Its purpose is to approach the problem with martingale techniques. The martingale prerequisites are given in $\S 2$. To obtain $(* *)$ we first prove $L^{1}$-estimations for certain square functions related to $h$ (see Lemma 4). These are derived using a "step-by-step" method (explained at the beginning of $\$ 5$ ) and an examination of what happens at each increment. More precisely, we have to consider at this point functions of the form $a+h-b \sigma$, where $a, b$ are scalars, $h \in H_{0}^{1}$ and $\sigma=$ sign cos.

Minorations of the $L^{-1}$-norm of such expressions are given in Propositions 8 and 9 below. It is only at this place that some complex function theory will be involved.

2. Martingale preliminaries. Let $\left(\mathscr{F}_{k}\right)_{k=0,1,2, \ldots}$ be an increasing sequence of $\sigma$ algebras on a probability space $(\Omega, \mathscr{F}, \mathbf{P})$ assuming $\mathscr{F}=\bigvee_{k=1}^{\infty} \mathscr{F}_{k}$. Denote by $\mathbf{E}_{k}$ the expectation with respect to $\mathscr{F}_{k}$. For $f \in L^{1}(\mathscr{F})$ let

$$
f^{*}=\sup _{k}\left|\mathbf{E}_{k}[f]\right| \text { and } S(f)=\left[\left|\mathbf{E}_{0}[f]\right|^{2}+\sum_{k=1}^{\infty}\left|\mathbf{E}_{k}[f]-\mathbf{E}_{k-1}[f]\right|^{2}\right]^{1 / 2} \text {. }
$$

We will use the notation $C$ to indicate a numerical constant. Let us recall the following result, due to D. Davis (see [7]).

Proposition 4. $C^{-1}\|S(f)\|_{1} \leqslant\left\|f^{*}\right\|_{1} \leqslant C\|S(f)\|_{1}$.

The next inequality is probably known, but we include its proof here for the sake of completeness.

Proposition 5. Let $\left(v_{k}\right)$ be an adapted sequence of functions; thus $v_{k}$ is $\mathscr{F}_{k}$ measurable for each $k$. Then

$$
\left\|\left[\sum\left|\mathbf{E}_{k-1}\left[\left|v_{k}\right|\right]\right|^{2}\right]^{1 / 2}\right\|_{1} \leqslant C\left\|\left[\sum\left|v_{k}\right|^{2}\right]^{1 / 2}\right\|_{1}
$$

Proof. It is no restriction to assume the $\mathscr{F}_{k}$ finite algebras. Moreover, since one may always tensor the $v_{k}$ against a Rademacher sequence, we can assume $\mathbf{E}_{k-1}\left[v_{k}\right]$ $=0$ and thus $\left(v_{k}\right)$ is an adapted martingale difference sequence. Since, then

$$
\left\|\left[\sum\left|v_{k}\right|^{2}\right]^{1 / 2}\right\|_{1}=\left\|\sum v_{k}\right\|_{H^{1}\left(\mathscr{F}_{k}\right)},
$$

it follows from the atomic decomposition property for $H^{1}$-functions (see for instance [7, Chapter I]) and convexity, that we may take for $\Sigma v_{k}$ a function of the form (for some positive integer $j$ )

$$
a=\frac{1}{|A|}\left(\varphi-\mathbf{E}_{j-1}[\varphi]\right)
$$

where $A$ is an $\mathscr{F}_{j}$-atom, supp $\varphi \subset A$ and $\|\varphi\|_{\infty} \leqslant 1$. In this case

$$
\begin{aligned}
v_{k} & =\mathbf{E}_{k}[a]-\mathbf{E}_{k-1}[a]=0 \quad \text { for } k<j, \\
& =\frac{1}{|A|}\left(\mathbf{E}_{k}[\varphi]-\mathbf{E}_{k-1}[\varphi]\right) \text { for } k \geqslant j
\end{aligned}
$$


Also, $\mathbf{E}_{k}[\varphi]$ is supported by $A$ for $k \geqslant j$ and hence $v_{k}$ for $k>j$. Thus the left side in Proposition 5 is dominated by

$$
\begin{gathered}
\left\|v_{j}\right\|_{1}+\left\|\left(\sum_{k>j} \mathbf{E}_{k-1}\left[\left|v_{k}\right|^{2}\right]\right)^{1 / 2}\right\|_{1} \\
\leqslant 2+\int_{A}\left(\sum_{k>j} \mathbf{E}_{k-1}\left[\left|v_{k}\right|^{2}\right]\right)^{1 / 2} \quad \text { (by Cauchy-Schwarz) } \\
\leqslant 2+|A|^{1 / 2}\left(\int \sum_{k>j}\left|v_{k}\right|^{2}\right)^{1 / 2} \\
\leqslant 2+|A|^{1 / 2}\|a\|_{2} \leqslant 3,
\end{gathered}
$$

proving the result.

Proposition 6. For $f \in H^{1}\left(\mathscr{F}_{k}\right)$, one has an inequality

$$
\left(\sum\left\|\left(\mathbf{E}_{k}-\mathbf{E}_{k-1}\right)[f]\right\|_{1}^{2}\right)^{1 / 2} \leqslant C\|f\|_{1}^{1 / 2}\|f\|_{H^{1}}^{1 / 2}
$$

To prove this, we will first deal with the special case of the Rademacher projection on the Cantor group (in fact, only this will be used later on).

Proposition 7. If $D=\{1,-1\}^{\mathbf{N}}$ is the Cantor-group and $f \in H^{1}(D)$, then

$$
\left(\sum|\hat{f}(k)|^{2}\right)^{1 / 2} \leqslant C\|f\|_{1}^{1 / 2}\|f\|_{H^{1}}^{1 / 2}
$$

where $\hat{f}(k)=\int f(\varepsilon) \varepsilon_{k}$.

Proof. We will use the theorem of [6] on the BMO-distance of a BMO-function to $L^{\infty}$ (in the diadic setting). The result asserts, in particular, that for $\varphi \in \operatorname{BMO}(D)$, $\operatorname{dist}_{\mathrm{BMO}}\left(\varphi, L^{\infty}\right)=0$ and $\varepsilon>0$, there exists a decomposition $\varphi=\alpha+\beta$ such that

$$
\|\alpha\|_{\mathrm{BMO}} \leqslant C_{1} \varepsilon \text { and }\|\beta\|_{\infty} \leqslant C_{2} \max \left(\varepsilon, \lambda_{0}(\varepsilon)\right)
$$

where $\lambda_{0}=\lambda_{0}(\varepsilon)$ has to satisfy

$$
\sup _{I} \frac{1}{|I|}\left|\left\{x \in I,\left|\varphi(x)-\varphi_{I}\right|>\lambda\right\}\right| \leqslant e^{-\lambda / \varepsilon}
$$

whenever $\lambda>\lambda_{0}\left(\varphi_{I}=|I|^{-1} \int_{I} \varphi\right)$.

Now take $\varphi=\sum a_{k} \varepsilon_{k}$ with $\sum\left|a_{k}\right|^{2}=1$. It follows from the distribution property of Rademacher that for each diadic interval $I$,

$$
\left|\left\{\alpha \in I ;\left|\varphi(x)-\varphi_{I}\right|>\lambda\right\}\right| \leqslant C e^{-c \lambda^{2}}|I|
$$

for numerical constants $c>0, C<\infty$. Hence $\operatorname{dist}_{\mathrm{BMO}}\left(\varphi, L^{\infty}\right)=0$ and $\lambda_{0}(\varepsilon) \sim 1 / \varepsilon$.

Decomposing $\varphi=\alpha+\beta$ as above, we get

$$
|\langle f, \varphi\rangle| \leqslant|\langle f, \alpha\rangle|+|\langle f, \beta\rangle| \leqslant C_{1}^{\prime} \varepsilon\|f\|_{H^{\prime}}+C_{2}^{\prime} \frac{1}{\varepsilon}\|f\|_{1} .
$$

Taking supremum over $\varphi$ and choosing $\varepsilon=\|f\|_{1}^{1 / 2}\|f\|_{H^{1}}^{-1 / 2}$, the inequality follows. 
Proof of Proposition 6. Assume $f$ real and estimate

$$
\left(\sum_{k=1}^{K}\left\|\left(\mathbf{E}_{k}-\mathbf{E}_{k-1}\right)[f]\right\|_{1}^{2}\right)^{1 / 2}
$$

Define for each $k$,

$$
\sigma_{k}=\operatorname{sign} \Delta f_{k} \text { and } b_{k}=\frac{1}{2}\left(\sigma_{k}-\mathbf{E}_{k-1}\left[\sigma_{k}\right]\right) .
$$

Then

$$
\|f\|_{1} \geqslant \iint|f| \prod_{k=1}^{K}\left(1+\varepsilon_{k} b_{k}\right) d \varepsilon \mathbf{P}(d \omega) \geqslant \frac{1}{2} \int\left|\sum_{k=1}^{K} \varepsilon_{k} \Phi_{k}(\varepsilon)\right| d \varepsilon
$$

where

$$
\Phi_{k}(\varepsilon)=\int \prod_{j=1}^{k-1}\left(1+\varepsilon_{j} b_{j}\right)\left|\Delta f_{k}\right| d \omega .
$$

Application of Proposition 7 to the function $\sum \varepsilon_{k} \Phi_{k}(\varepsilon)$ then gives

$$
\begin{aligned}
\left(\sum_{k=1}^{K}\left\|\Delta f_{k}\right\|_{1}^{2}\right)^{1 / 2} & \leqslant C\|f\|_{1}^{-1 / 2}\left[\int\left(\sum\left|\phi_{k}(\varepsilon)\right|^{2}\right)^{1 / 2} d \varepsilon\right]^{1 / 2} \\
& \leqslant C\|f\|_{1}^{1 / 2}\left[\iint S(f) \Pi\left(1+\varepsilon_{j} b_{j}\right) d \omega d \varepsilon\right]^{1 / 2} \\
& =C\|f\|_{1}^{1 / 2}\|f\|_{H^{1}}^{1 / 2}
\end{aligned}
$$

as announced.

REMARK. The author is grateful to P. W. Jones for outlining a more explicit procedure to obtain the decomposition used in the proof of Proposition 7.

3. Some inequalities involving $H_{0}^{1}$-functions. The purpose of this section is to prove the following results.

Proposition 8. For $a \in \mathbf{C}$ and $h \in H_{0}^{1}$, one has

$$
\|a+h\|_{1} \geqslant\left\|\left(|a|^{2}+\delta^{2}|h|^{2}\right)^{1 / 2}\right\|_{1}
$$

where $\delta>0$ is a fixed constant.

Proposition 9. There exists $\delta>0$ such that for $a \in \mathbf{C}, b \in \mathbf{C}$ and $h \in H_{0}^{1}$,

$$
\|a+h-b \sigma\|_{1} \geqslant\left\{|a|^{2}+\delta^{2}\left[\frac{\operatorname{Re}(\overline{\langle h, \sigma\rangle}(\langle h, \sigma\rangle-b))}{|\langle h, \sigma\rangle|+|b|}\right]^{2}\right\}^{1 / 2},
$$

$$
\|a+h-\langle h, \sigma\rangle \sigma\|_{1} \geqslant\left\|\left\{|a|^{2}+\delta^{2}\left|h_{e}-\langle h, \sigma\rangle \sigma\right|^{2}\right\}^{1 / 2}\right\|_{1}
$$

where $\sigma=\operatorname{sign} \cos$ and $h_{e}(\theta)=\sum_{n=1}^{\infty} \hat{h}(n) \cos n \theta$. 
It is clear that it suffices to prove Propositions 8 and 9, with $a=1$.

Proof of Proposition 8. Factoring $1+h$ gives $1+h=\left(1+g_{1}\right)\left(1+g_{2}\right)$ where $g_{1}, g_{2} \in H_{0}^{2}$ and

$$
\|1+h\|_{1}=\left(1+\left\|g_{1}\right\|_{2}^{2}\right)^{1 / 2}\left(1+\left\|g_{2}\right\|_{2}^{2}\right)^{1 / 2} .
$$

Since $|h| \leqslant\left|g_{1}\right|+\left|g_{2}\right|+\left|g_{1}\right|\left|g_{2}\right|$ the result follows from the majorations

$$
\left\|\left(1+\left|g_{i}\right|^{2}\right)^{1 / 2}\right\|_{1} \leqslant\left\|\left(1+\left|g_{i}\right|^{2}\right)^{1 / 2}\right\|_{2}=\left(1+\left\|g_{i}\right\|_{2}^{2}\right)^{1 / 2} \leqslant\|1+h\|_{1} \quad(i=1,2)
$$

and

$$
\left\|\left(1+\left|g_{1}\right|^{2}\left|g_{2}\right|^{2}\right)^{1 / 2}\right\|_{1} \leqslant 1+\left\|g_{1} g_{2}\right\|_{1} \leqslant 1+\left\|g_{1}\right\|_{2}\left\|g_{2}\right\|_{2} \leqslant\|1+h\|_{1} .
$$

Also to obtain Proposition 9, we will use the $L^{2}$-theory. Our argument here is, however, more complicated. This is the only point where explicit constructions of $H^{\infty}$-functions appear.

Lemma 1. Given a measurable subset $A$ of $\Pi$, there exist $H^{\infty}$-functions $\varphi$ and $\psi$ satisfying the following conditions:

(i) $|\varphi|+|\psi| \leqslant 1$,

(ii) $\operatorname{Re} \psi$ is an even function on $\Pi$,

(iii) $|\varphi-1 / 8|<1 / 100$ on the set $A$,

(iv) $\|\varphi\|_{1} \leqslant C|A|$,

(v) $\|\operatorname{Re} \psi-1\|_{1} \leqslant C|A|$.

Proof. Fix some (large) $M>0$ and define the following $H^{\infty}$-functions:

$$
\begin{gathered}
\tau(z)=-M \int_{A} \frac{e^{i \theta}+z}{e^{i \theta}-z} m(d \theta), \quad \varphi=\frac{1}{8}\left(1-e^{\tau}\right)^{2}, \\
\psi(z)=\exp \left\{\int_{\Pi} \log (1-\alpha(\theta)) \frac{e^{i \theta}+z}{e^{i \theta}-z} m(d \theta)\right\}
\end{gathered}
$$

where $\alpha(\theta)=\left|\varphi\left(e^{i \theta}\right)\right| \vee\left|\varphi\left(e^{-i \theta}\right)\right|$.

Notice that this makes sense, because $e^{\tau}$ has boundary value $e^{-M\left(\chi_{A}+i \mathcal{H}\left(\chi_{A}\right)\right)}$ ( $\mathcal{H}=$ Hilbert-transform) and therefore $\|\alpha\|_{\infty} \leqslant \frac{1}{2}$.

(i) is obvious. On $\Pi$, we have $\operatorname{Re} \psi=(1-\alpha) \cos \mathcal{H}(\log (1-\alpha))$ and thus an even function. Since $\left|\varphi-\frac{1}{8}\right| \leqslant \frac{3}{8}\left|e^{\tau}\right|$ and thus $\left|\varphi-\frac{1}{8}\right|<e^{-M}$ on $A$ (iii) holds for $M$ large enough. Because on $\Pi$

$$
8|\varphi| \leqslant \chi_{A}+M^{2}\left|\mathcal{H C}\left(\chi_{A}\right)\right|^{2}
$$

(iv) follows. Finally,

$$
|1-\operatorname{Re} \psi| \leqslant|\alpha|+\frac{1}{2}|\mathcal{H}(\log (1-\alpha))|^{2}, \quad\|1-\operatorname{Re} \psi\|_{1} \leqslant 4\|\varphi\|_{1}
$$

and hence (v).

We refer the reader to [4, Proposition 1.6] for the following Marcinkiewicz type decomposition. 
LEMMA 2. There is a constant $C<\infty$ such that for given $h \in H_{0}^{1}$ and $\lambda>0$, there exists $h_{\lambda} \in H_{0}^{\infty}$ satisfying:

(i) $\left|h_{\lambda}\right| \leqslant C|h|$,

(ii) $\left\|h_{\lambda}\right\|_{\infty} \leqslant C \lambda$,

(iii) $\left\|h-h_{\lambda}\right\|_{1} \leqslant C \int_{[|h|>\lambda]}|h|$.

Let $h$ be as in Proposition 9. For $\lambda>0$, define $A_{\lambda}=[|h|>\lambda]$. Application of Lemma 1 to the set $A_{\lambda}$ provides $H^{\infty}$-functions $\varphi_{\lambda}, \psi_{\lambda}$. We are now ready to prove

LEMMA 3. $\|1+h-b \sigma\|_{1} \geqslant 1+c \int_{A_{\lambda}}|h|+c \lambda^{-2}\left\|\operatorname{Im}\left(h_{\lambda}-b \sigma\right)\right\|_{2}^{2}$ if $\lambda>K$ and $|b|$ $<\lambda / K(c>0$ and $K<\infty$ being numerical constants $)$.

Proof. First, since $1-b \sigma$ is even and $\operatorname{Im} \psi_{\lambda}$ odd, we find

$$
\begin{aligned}
\|1+h-b \sigma\|_{1} & \geqslant\left\|(1+h-b \sigma) \varphi_{\lambda}\right\|_{1}+\left|\int(1-b \sigma) \psi_{\lambda}\right| \\
& \geqslant \frac{1}{9} \int_{A_{\lambda}}|| h|-(1+|b|)|+\left|\int(1-b \sigma) \operatorname{Re} \psi_{\lambda}\right| \\
& \geqslant \frac{1}{9} \int_{A_{\lambda}}|h|-\frac{1}{9}(1+|b|)\left|A_{\lambda}\right|+1-(1+|b|)\left\|1-\operatorname{Re} \psi_{\lambda}\right\|_{1} \\
& \geqslant \frac{1}{9} \int_{A_{\lambda}}|h|-C(1+|b|)\left|A_{\lambda}\right|+1
\end{aligned}
$$

for some constant $C$. Thus, choosing $K$ large enough, we get

$$
\|1+h-b \sigma\|_{1} \geqslant 1+\frac{1}{10} \int_{A_{\lambda}}|h| .
$$

Fix some small constant $\delta>0$. Since we always have

$$
\|1+a f\|_{1} \leqslant\|1+f\|_{1} \text { for } 0 \leqslant a \leqslant 1 \text { and } f \text { of mean } 0,
$$

it follows that

$$
\|1+h-b \sigma\|_{1} \geqslant\left\|1+\delta \lambda^{-1}(h-b \sigma)\right\|_{1} \geqslant\left\|1+\delta \lambda^{-1}\left(h_{\lambda}-b \sigma\right)\right\|_{1}-\delta \lambda^{-1}\left\|h-h_{\lambda}\right\|_{1} .
$$

Because $\delta \lambda^{-1}\left|h_{\lambda}-b \sigma\right| \ll 1$ the inequality

$$
(1+t)^{1 / 2} \geqslant 1+t / 3 \text { for } 0 \leqslant t \leqslant 1
$$

yields

$$
\left|1+\delta \lambda^{-1}\left(h_{\lambda}-b \sigma\right)\right| \geqslant\left[1+\delta \lambda^{-1} \operatorname{Re}\left(h_{\lambda}-b \sigma\right)\right]\left[1+\frac{1}{12} \delta^{2} \lambda^{-2}\left(\operatorname{Im}\left(h_{\lambda}-b \sigma\right)\right)^{2}\right] .
$$

Therefore, also

$$
\|1+h-b \sigma\|_{1} \geqslant 1+\frac{1}{20} \delta^{2} \lambda^{-2} \int \operatorname{Im}^{2}\left(h_{\lambda}-b \sigma\right)-c \delta \lambda^{-1} \int_{A_{\lambda}}|h| .
$$

The required minoration clearly follows combining $(*)$ and $(* *)$.

Proof of Proposition 9. First

$$
\|1+h-b \sigma\|_{1} \geqslant d\left(b \sigma, H^{1}\right) \geqslant \frac{|b|}{2 \pi}\left|\int_{-\pi}^{\pi} \sigma(\theta) e^{i \theta} d \theta\right|=\frac{2}{\pi}|b|
$$


and hence, also,

$$
\|1+h-b \sigma\|_{1} \geqslant \frac{1}{3}\|1+h\|_{1} \geqslant \frac{1}{3}\|h\|_{1} .
$$

Notice that the right member of (i), (ii) is bounded by $1+2 \delta\|h\|_{1}$. Since now $\|1+h-b \sigma\|_{1} \geqslant \frac{1}{6}\|h\|_{1}+\frac{1}{6}|b|$, it follows that (i) (resp. (ii)) are satisfied for $|b| \geqslant 6$ (resp. $|\langle h, \sigma\rangle| \geqslant 6$ ). Hence, we may assume $|b| \leqslant M$ in (i), $|\langle h, \sigma\rangle| \leqslant M$ in (ii) where $M$ is some numerical constant.

Fix a constant $\lambda>K M$ and put $k=h_{\lambda}$ for simplicity. Using again Lemma 2(iii), the right member of (i) can be majorized by

$$
\begin{aligned}
& {\left[1+2 \delta^{2}\left(|\operatorname{Re}\langle h, \sigma\rangle|^{2}+|\operatorname{Im}(\langle h, \sigma\rangle-b)|^{2}\right)\right]^{1 / 2} } \\
& \leqslant\left[1+2 \delta^{2}\left(|\operatorname{Re}\langle k, \sigma\rangle|^{2}+|\operatorname{Im}(\langle k, \sigma\rangle-b)|^{2}\right)\right]^{1 / 2}+2 \delta C \int_{A_{\lambda}}|h| .
\end{aligned}
$$

Taking Lemma 3 into account, we see that it suffices to check the inequality

$$
|\operatorname{Re}\langle k, \sigma\rangle|^{2}+|\operatorname{Im}(\langle k, \sigma\rangle-b)|^{2} \leqslant\|\operatorname{Im}(k-b \sigma)\|_{2}^{2}
$$

which is straightforward:

$$
\|\operatorname{Im}(k-b \sigma)\|_{2}^{2}=\frac{1}{2} \sum_{n>0}|\operatorname{Im} \hat{k}(n)-2 \operatorname{Im} b \hat{\sigma}(n)|^{2}+\frac{1}{2} \sum_{n>0}|\operatorname{Re} \hat{k}(n)|^{2}
$$

while

$$
\begin{aligned}
|\operatorname{Re}\langle k, \sigma\rangle| \leqslant \sum_{n>0}|\operatorname{Re} \hat{k}(n)| \hat{\boldsymbol{\sigma}}(n) \leqslant \frac{1}{\sqrt{2}}\left(\sum|\operatorname{Re} \hat{k}(n)|^{2}\right)^{1 / 2}, \\
|\operatorname{Im}(\langle k, \sigma\rangle-b)| \leqslant \sum_{n>0}|\operatorname{Im} \hat{k}(n)-2 \operatorname{Im} b \hat{\boldsymbol{\sigma}}(n)| \hat{\boldsymbol{\sigma}}(n) \\
\leqslant \frac{1}{\sqrt{2}}\left(\sum_{n>0}|\operatorname{Im} \hat{k}(n)-2 \operatorname{Im} b \hat{\boldsymbol{\sigma}}(n)|^{2}\right)^{1 / 2} .
\end{aligned}
$$

For the right member of (ii), a similar reasoning reduces the question to the verification of

$$
\int\left|k_{e}-\langle k, \sigma\rangle \sigma\right|^{2} \leqslant\|\operatorname{Im}(k-b \sigma)\|_{2}^{2}
$$

which the reader will easily do.

4. Reduction of the problem. In this section, we will reduce the problem of proving that certain elements of $L^{1}(\Pi)$ normed by the quotient norm $L^{1} / H^{1}$ to the verification of an inequality for certain functions in $L^{1}\left(\Pi^{\mathbf{N}}\right)$, where $\Pi^{\mathbf{N}}=\Pi \times \Pi \times \cdots$ is the product group. Denote by $\mathbf{E}_{k}(k=1,2, \ldots)$ the expectation with respect to the $k$ first variables $\left(x_{1}, x_{2}, \ldots, x_{k}\right)$, where $x=\left(x_{1}, x_{2}, \ldots\right)$ is the product variable.

We consider the subspace $\mathcal{H}$ of $L^{1}\left(\Pi^{\mathbf{N}}\right)$ of those functions $h$ such that for each $k$ the difference $\mathbf{E}_{k}[h]-\mathbf{E}_{k-1}[h]$ is an $H_{0}^{1}$-function with respect to $x_{k}$. Thus $h$ is of the form

$$
h=\sum h_{k} \quad \text { where } h_{k}=\sum_{n>0} \hat{h}_{k}(n) e^{i n x_{k}}
$$

and the $\hat{h}_{k}(n)$ are functions of $x_{1}, \ldots, x_{k-1}$. 
Again let $\sigma=\operatorname{sign} \cos$ and $\sigma_{k}(x)=\sigma\left(x_{k}\right)$ for each $k$. Let $\mathscr{F}$ be the $\sigma$-algebra on $\Pi^{N}$ generated by the $\sigma_{k}$. In the next section, we show the following

Proposition 10. There is a constant $c>0$ s.t. $\left\|h-\mathbf{E}_{\mathscr{F}}[h]\right\|_{1} \geqslant c\|h\|_{1}$ for all $h \in \mathcal{K}$.

This fact obviously implies

COROLlaRY 11. $\inf _{h \in \mathcal{C}}\|f-h\|_{1} \geqslant c^{\prime}\|f\|_{1}$ for all $f \in L^{1}(\mathscr{F})$.

For $a, n$ positive integers, $\mathscr{F}_{a}$ will be the Fejér kernel

$$
F_{a}(\theta)=\sum_{|j| \leqslant a} \frac{a+1-|j|}{a+1} e^{i j \theta}
$$

and $F_{a, n}(\theta)=F_{a}(n \theta)$.

We consider sequences of positive integers $\left(n_{k}\right),\left(a_{k}\right)$ satisfying the following conditions: ( $\varsigma$ denotes again the $\sigma$-algebra on II generated by the functions $\sigma\left(n_{k} \theta\right)$.)

(i) The transference property, i.e. let $E=\left\{\Sigma^{\prime} \nu_{k} n_{k} ;\left(\nu_{k}\right) \in F\right\}$ where $F$ is the subset $\left\{\left(\nu_{k}\right),\left|\nu_{k}\right| \leqslant a_{k}\right\}$ of the dual group of $\Pi^{\mathbf{N}}$. Then the operator

$$
T: L_{E}^{1}(\Pi) \rightarrow L_{F}^{1}\left(\Pi^{n}\right), \quad T(f)(x)=\sum_{\left(\nu_{k}\right) \in F} \hat{f}\left(\sum \nu_{k} n_{k}\right) e^{i\left(\sum \nu_{k} x_{k}\right)}
$$

satisfies

$$
\frac{1}{2}\|f\|_{1} \leqslant\|T(f)\|_{1} \leqslant 2\|f\|_{1} .
$$

Moreover, $T(f) \in \mathcal{H}$ for $f \in L_{E}^{1} \cap H_{0}^{1}$.

(ii) Defining for each $k$,

$$
\begin{gathered}
\xi_{k}=\sigma * F_{a_{k}}, \quad K=\prod_{k} F_{a_{k}, n_{k}}, \\
R(\theta, \psi)=\prod\left[1+\xi_{k}\left(n_{k} \theta\right) \sigma\left(n_{k} \psi\right)\right],
\end{gathered}
$$

one has

( $\alpha) \sum\left\|\xi_{k}-\sigma\right\|_{1} \leqslant \varepsilon$

( $\beta)\|K\|_{1}=1$.

For $f \in L^{1}(\Im)$,

$(\gamma)\|f-f * K\|_{1}<\varepsilon\|f\|_{1}$,

( $\delta)\|f-R(f)\|_{1}<\varepsilon\|f\|_{1}$ where $R(f)(\theta)=\int f(\psi) R(\theta, \psi) m(d \psi)$ (where $\varepsilon>0$ is a small constant).

The reader will easily convince himself that the realisation of these conditions is straightforward. Details on the transference property can be found in [1].

Let us now show that the sequence $\left(n_{k}\right)$ satisfies the Theorem. Thus, fix $f \in L^{1}(\Im)$ and $h \in H_{0}^{1}$. We get, by (ii),

$$
\|f-h\|_{1} \geqslant\|f * K-h * K\|_{1} \geqslant\|R(f)-h * K\|_{1}-2 \varepsilon\|f\|_{1} .
$$

Notice that $R(f) \in L_{E}^{1}$. By (i),

$$
\|R(f)-h * K\|_{1} \geqslant \frac{1}{2}\left\|T(R(f))-h_{1}\right\|_{1}
$$

vhere $h_{1}=T(h * K) \in \mathcal{H}$. 
Now

$$
T(R(f))(x)=\int f(\psi) \prod\left[1+\xi_{k}\left(x_{k}\right) \sigma\left(n_{k} \psi\right)\right] m(d \psi) .
$$

By (ii) $(\alpha)$, we see that for any $( \pm 1)$-sequence $\left(\tau_{k}\right)$

$$
\left\|\otimes\left(1+\tau_{k} \xi_{k}\right)-\Pi\left(1+\tau_{k} \sigma_{k}\right)\right\|_{1}<\varepsilon
$$

implying that

$$
\left\|T(R(f))-f_{1}\right\| \leqslant 2 \varepsilon\|f\| \quad \text { where } f_{1}=\mathbf{E}[T(R(f))] .
$$

It follows then from Corollary 11 that

$$
\begin{aligned}
\|f-h\|_{1} & \geqslant \frac{1}{2}\left\|f_{1}-h_{1}\right\|_{1}-3 \varepsilon\|f\|_{1} \geqslant \frac{c}{2}\left\|f_{1}\right\|_{1}-3 \varepsilon\|f\|_{1} \\
& \geqslant \frac{c}{2}\|T(R(f))\|_{1}-4 \varepsilon\|f\|_{1} \geqslant \frac{c}{4}\|f\|_{1}-5 \varepsilon\|f\|_{1} \geqslant c^{\prime}\|f\|_{1}
\end{aligned}
$$

taking $\varepsilon>0$ small enough.

5. Proof of the Theorem. It remains to prove Proposition 10. So fix $h=\sum h_{k} \in \mathcal{H}$ where

$$
h_{k}=\sum_{n>0} \hat{h}_{k}(n)\left(x_{1}, \ldots, x_{k-1}\right) e^{i n x_{k}}
$$

We also define

$$
\begin{aligned}
{\left[h_{k}\right]_{e} } & =\sum \hat{h}_{k}(n) \cos n x_{k}, \\
{\left[h_{k}\right]_{0} } & =\sum \hat{h}_{k}(n) \sin n x_{k}, \\
\left\langle h_{k}, \sigma_{k}\right\rangle & =\sum \hat{h}_{k}(n) \hat{\sigma}(\eta)
\end{aligned}
$$

(which is thus a function of $x_{1}, \ldots, x_{k-1}$ ). If $f=\mathbf{E}_{\mathscr{F}}[h]$, then $f=\sum b_{k} \cdot \sigma_{k}$, where $b_{k}=b_{k}\left(x_{1}, \ldots, x_{k-1}\right)=\mathbf{E}_{\mathscr{F}}\left[\left\langle h_{k}, \sigma_{k}\right\rangle\right]$.

Using E. Stein's theorem on $H^{1}$-multipliers (see [11]), it is easily seen that $\|h\|_{1} \sim\|S(h)\|_{1}(S=$ square function with respect to the natural decomposition).

We give a direct proof of this fact, based on Proposition 8 .

Fix $1>\varepsilon>0$ and a positive sequence $\left(s_{k}\right)_{k=1,2, \ldots}$ in $L^{\infty}\left(\Pi^{\mathbf{N}}\right)$ satifying $\left\|\left(\sum s_{k}^{2}\right)^{1 / 2}\right\|_{\infty} \leqslant \varepsilon$. Fixing a positive integer $K$, we get, using Proposition 8 ,

$$
\begin{aligned}
\left\|\mathbf{E}_{K}[h]\right\|_{1} & =\left\|\mathbf{E}_{K-1}[h]+h_{K}\right\|_{1} \\
& \geqslant\left\|\left(\left|\mathbf{E}_{K-1}[h]\right|^{2}+\delta^{2}\left|h_{K}\right|^{2}\right)^{1 / 2}\right\|_{1} \\
& \geqslant\left\|\left|\mathbf{E}_{K-1}[h]\right|\left(1-s_{K}^{2}\right)^{1 / 2}\right\|_{1}+\delta\left\|\left|h_{K}\right| s_{K}\right\|_{1} \\
& \geqslant\left\|\mathbf{E}_{K-1}[h]\right\|_{1}+\delta\left\|\left|h_{K}\right| s_{K}\right\|_{1}-\left\|\mathbf{E}_{K-1}[h] s_{K}^{2}\right\|_{1} .
\end{aligned}
$$

Iterating,

$$
\begin{aligned}
\|h\|_{1} & \geqslant \delta \sum\left\|\left|h_{k}\right| s_{k}\right\|_{1}-\sum\left\|\mathbf{E}_{k-1}[h] s_{k}^{2}\right\|_{1} \\
& \geqslant \delta \sum\left\|\left|h_{k}\right| s_{k}\right\|_{1}-\varepsilon^{2}\left\|\max _{k}\left|\mathbf{E}_{k}[h]\right|\right\|_{1} .
\end{aligned}
$$


Taking supremum over the sequences $\left(s_{k}\right)$, it follows that

$$
\|h\|_{1} \geqslant \delta \varepsilon\|S(h)\|_{1}-\varepsilon^{2}\left\|\max _{k}\left|\mathbf{E}_{k}[h]\right|\right\|_{1}
$$

and choosing

$$
\varepsilon^{2}=\frac{\|h\|_{1}}{\left\|\max \left|\mathbf{E}_{k}[h]\right|\right\|_{1}}
$$

we get

$$
\|S(h)\|_{1} \leqslant \delta^{-1}\|h\|_{1}^{1 / 2}\left\|\max \left|\mathbf{E}_{k}[h]\right|\right\|_{1}^{1 / 2} .
$$

Hence, by Proposition 4, $\|S(h)\|_{1} \leqslant \delta^{-2}\|h\|_{1}$ as required.

Before continuing, notice that since $\mathscr{F}$-expectation is a contraction, $\|S(f)\|_{1} \leqslant$ $\|S(h)\|_{1}$. Since for each $k,|\cdots|\left\langle h_{k}, \sigma_{k}\right\rangle \mid \leqslant \mathbf{E}_{k-1}\left[\left|h_{k}\right|\right]$, application of Proposition 5 yields

$$
\left\|\left(\sum\left|\left\langle h_{k}, \sigma_{k}\right\rangle\right|^{2}\right)^{1 / 2}\right\|_{1} \leqslant C\|h\|_{1} .
$$

If we now apply the previous procedure using Proposition 9, the following inequalities are derived.

\section{LEMMA 4.}

$$
\left\|\left\{\sum_{k}\left|\frac{\operatorname{Re} \overline{\left\langle h_{k}, \sigma_{k}\right\rangle}\left(\left\langle h_{k}, \sigma_{k}\right\rangle-b_{k}\right)}{\left|\left\langle h_{k}, \sigma_{k}\right\rangle\right|+\left|b_{k}\right|}\right|^{2}\right\}^{1 / 2}\right\|_{1} \leqslant C\|h-f\|_{1}^{1 / 2}\|h\|_{1}^{1 / 2},
$$

$$
\left\|\left\{\sum_{k}\left|\left[h_{k}\right]_{8}-\left\langle h_{k}, \sigma_{k}\right\rangle \sigma_{k}\right|^{2}\right\}^{1 / 2}\right\|_{1} \leqslant C\left\|h-\sum\left\langle h_{k}, \sigma_{k}\right\rangle \sigma_{k}\right\|_{1}^{1 / 2}\|h\|_{1}^{1 / 2} .
$$

Proof. Let us show how (I) follows from Proposition 9(i). The argument for (II) is analogous. Fix $0<\varepsilon<1$ and a sequence $\left(s_{k}\right)_{k=1,2, \ldots}$ of positive $L^{\infty}$-functions on $\Pi^{\mathbf{N}}$ satisfying $\left\|\left(\sum s_{k}^{2}\right)^{1 / 2}\right\|_{\infty} \leqslant \varepsilon$. Fix an integer $k$ and apply Proposition 9(i) in the variable $x_{k}$. We get

$$
\begin{aligned}
\left\|\mathbf{E}_{k}[h-f]\right\|_{1}= & \left\|\mathbf{E}_{k-1}[h-f]+h_{k}-b_{k} \sigma_{k}\right\|_{1} \\
& \geqslant\left\|\left\{\left|\mathbf{E}_{k-1}[h-f]\right|^{2}+\delta^{2}\left[\frac{\operatorname{Re} \overline{\left\langle h_{k}, \sigma_{k}\right\rangle}\left(\left\langle h_{k}, \sigma_{k}\right\rangle-b_{k}\right)}{\left|\left\langle h_{k}, \sigma_{k}\right\rangle\right|+\left|b_{k}\right|}\right]^{2}\right\}^{1 / 2}\right\|_{1} \\
\geqslant & \left\|\mathbf{E}_{k-1}[h-f]\right\|_{1}+\delta\left\|\frac{\operatorname{Re} \overline{\left\langle h_{k}, \sigma_{k}\right\rangle}\left(\left\langle h_{k}, \sigma_{k}\right\rangle-b_{k}\right)}{\left|\left\langle h_{k}, \sigma_{k}\right\rangle\right|+\left|b_{k}\right|} s_{k}\right\|_{1} \\
& -\left\|\mathbf{E}_{k-1}[h-f] s_{k}^{2}\right\|_{1} .
\end{aligned}
$$

Iterating and using the same considerations as in the beginning of this section it follows that the left member of (I) is dominated by

$$
\delta^{-1} \varepsilon^{-1}\|h-f\|_{1}+\text { const. } \varepsilon\|S(h-f)\|_{1},
$$


and hence, choosing $\varepsilon$ appropriately, by the right member of (I). We first make use of (I) to show

LEMMA 5. $\left\|\left[\sum\left|\left\langle h_{k}, \sigma_{k}\right\rangle-b_{k}\right|^{2}\right]^{1 / 2}\right\|_{1} \leqslant C\|h-f\|_{1}^{1 / 4}\|h\|_{1}^{3 / 4}$.

Proof. Write

$$
2 \frac{\operatorname{Re} \overline{\left\langle h_{k}, \sigma_{k}\right\rangle}\left(\left\langle h_{k}, \sigma_{k}\right\rangle-h_{k}\right)}{\left|\left\langle h_{k}, \sigma_{k}\right\rangle\right|+\left|b_{k}\right|}=\xi_{k}-\left|b_{k}\right|
$$

where

$$
\xi_{k}=\frac{\left|\left\langle h_{k}, \sigma_{k}\right\rangle-b_{k}\right|^{2}}{\left|\left\langle h_{k}, \sigma_{k}\right\rangle\right|+\left|b_{k}\right|}+\left|\left\langle h_{k}, \sigma_{k}\right\rangle\right|
$$

By the triangle inequality, the left side of (I) dominates

$$
\left\|\left(\sum\left|\xi_{k}\right|^{2}\right)^{1 / 2}\right\|_{1}-\left\|\left(\sum\left|b_{k}\right|^{2}\right)^{1 / 2}\right\|_{1}
$$

Also, since $b_{k}=\mathbf{E}\left[\left\langle h_{k}, \sigma_{k}\right\rangle\right]$,

$$
\left\|\left(\sum\left|b_{k}\right|^{2}\right)^{1 / 2}\right\|_{1} \leqslant\left\|\left(\sum\left|\left\langle h_{k}, \sigma_{k}\right\rangle\right|^{2}\right)^{1 / 2}\right\|_{1}
$$

Write

$$
\begin{aligned}
& {\left[\sum\left(\xi_{k}^{2}-\left|\left\langle h_{k}, \sigma_{k}\right\rangle\right|^{2}\right)\right]^{1 / 2}} \\
& \quad=\left[\left(\sum \xi_{k}^{2}\right)^{1 / 2}+\left(\sum\left|\left\langle h_{k}, \sigma_{k}\right\rangle\right|^{2}\right)^{1 / 2}\right]^{1 / 2}\left[\left(\sum \xi_{k}^{2}\right)^{1 / 2}-\left(\sum\left|\left\langle h_{k}, \sigma_{k}\right\rangle\right|^{2}\right)^{1 / 2}\right]^{1 / 2}
\end{aligned}
$$

and apply Cauchy-Schwarz. From (I) and previous observations

$$
\left\|\left[\sum\left(\xi_{k}^{2}-\left|\left\langle h_{k}, \sigma_{k}\right\rangle\right|^{2}\right)\right]^{1 / 2}\right\|_{1} \leqslant C\|h\|_{1}^{1 / 2}\|h-f\|_{1}^{1 / 4}\|h\|_{1}^{1 / 4}=C\|h-f\|_{1}^{1 / 4}\|h\|_{1}^{3 / 4} \text {. }
$$

Since for each $k$,

$$
\xi_{k}^{2}-\left|\left\langle h_{k}, \sigma_{k}\right\rangle\right|^{2}=\left(\xi_{k}+\left|\left\langle h_{k}, \sigma_{k}\right\rangle\right|\right) \frac{\left|\left\langle h_{k}, \sigma_{k}\right\rangle-b_{k}\right|^{2}}{\left|\left\langle h_{k}, \sigma_{k}\right\rangle\right|+\left|b_{k}\right|} \geqslant C\left|\left\langle h_{k}, \sigma_{k}\right\rangle-b_{k}\right|^{2} .
$$

Lemma 5 is proved.

The left side of Lemma 5 dominates $\left\|f-\Sigma\left\langle h_{k}, \sigma_{k}\right\rangle \sigma_{k}\right\|_{1}$.

LEMMA 6. $\left\|\Sigma\left[h_{k}\right]_{0}\right\|_{1}$ and $\left\|\left[\sum\left|\left[h_{k}\right]_{e}-b_{k} \sigma_{k}\right|^{2}\right]^{1 / 2}\right\|_{1} \leqslant C\|h-f\|_{1}^{1 / 8}\|h\|_{1}^{7 / 8}$.

Proof. Since $\Sigma\left[h_{k}\right]_{0}=h-\Sigma\left[h_{k}\right]_{e}$, the first inequality is a consequence of the second. Write

$$
\begin{aligned}
\left\|\left[\sum\left|\left[h_{k}\right]_{e}-b_{k} \sigma_{k}\right|^{2}\right]^{1 / 2}\right\|_{1} \leqslant & \left\|\left[\sum\left|\left[h_{k}\right]_{e}-\left\langle h_{k}, \sigma_{k}\right\rangle \sigma_{k}\right|^{2}\right]^{1 / 2}\right\|_{1} \\
& +\left\|\left[\sum\left|\left\langle h_{k}, \sigma_{k}\right\rangle-b_{k}\right|^{2}\right]^{1 / 2}\right\|_{1}
\end{aligned}
$$


which by Lemmas 4(II) and 5 is estimated by

$$
C\left\|h-\sum\left\langle h_{k}, \sigma_{k}\right\rangle \sigma_{k}\right\|_{1}^{1 / 2}\|h\|_{1}^{1 / 2}+C\|h-f\|_{1}^{1 / 4}\|h\|_{1}^{3 / 4} \leqslant C\|h-f\|_{1}^{1 / 8}\|h\|_{1}^{7 / 8} .
$$

Define for $u \in L^{1}\left(\Pi^{\mathbf{N}}\right)$,

$$
(u)_{e}(x)=\int_{D} u\left(\varepsilon_{1} x_{1}, \varepsilon_{2} x_{2}, \ldots\right) d \varepsilon
$$

( $=$ the natural projection on the even part in $\left.x_{1}, x_{2}, \ldots\right)$.

LemMA 7. $\left\|\left[\Sigma_{k}\left|\left(\hat{h}_{k}(1)\right)_{e}\right|^{2}\right]^{1 / 2}\right\|_{1} \leqslant C\|h-f\|_{1}^{11 / 16}\|h\|_{1}^{15 / 16}$.

Proof. At this point, we will make use of Proposition 7. Fix $x \in \Pi^{\mathbf{N}}$ and remark that the sequence of functions in $\varepsilon \in D$,

$$
\left[h_{k}\right]_{0}\left(\varepsilon_{1} x_{1}, \varepsilon_{2} x_{2}, \ldots\right),
$$

is a martingale difference sequence.

Moreover, the $k$ th Rademacher coefficient is clearly given by

$$
\sum_{n>0}\left(\hat{h}_{k}(n)\right)_{e}(x) \sin n x_{k}
$$

and Proposition 7 yields

$$
\begin{aligned}
& {\left[\sum_{k}\left|\sum_{n>0}\left(\hat{h}_{k}(n)\right)_{e}(x) \sin n x_{k}\right|^{2}\right]^{1 / 2}} \\
& \leqslant C\left[\int\left|\sum\left[h_{k}\right]_{0}(\varepsilon \cdot x)\right| d \varepsilon\right]^{1 / 2}\left[\int\left[\sum\left|\left[h_{k}\right]_{0}(\varepsilon \cdot x)\right|^{2}\right]^{1 / 2} d \varepsilon\right]^{1 / 2} .
\end{aligned}
$$

Integration in $x$, application of Cauchy-Schwarz and Lemma 6, gives

$$
\text { (+) }\left\|\left[\sum_{k}\left|\sum_{n>0}\left(\hat{h}_{k}(n)\right)_{e} \sin n x_{k}\right|^{2}\right]^{1 / 2}\right\| \leqslant C\|h-f\|_{1}^{1 / 16}\|h\|_{1}^{7 / 16}\left\|\left[\sum\left|\left[h_{k}\right]_{0}\right|^{2}\right]^{1 / 2}\right\|_{1}^{1 / 2} \text {. }
$$

Also

$$
\left\|\left[\sum\left|\left[h_{k}\right]_{0}\right|^{2}\right]^{1 / 2}\right\|_{1} \leqslant C\|h\|_{1} .
$$

On the other hand, we can multiply the $k$ th increment in the left member of $(+)$ by $\sin x_{k}$ and then take $\mathbf{E}_{k-1}$-expectation. Proposition 5 shows that

$$
\left\|\left[\sum_{k}\left|\left(\hat{h}_{k}(1)\right)_{e}\right|^{2}\right]^{1 / 2}\right\|_{1} \leqslant C\|h-f\|_{1}^{1 / 16}\|h\|_{1}^{15 / 16}
$$

proving Lemma 7.

Now, rewriting

$$
\left[\sum\left|\left[h_{k}\right]_{e}-b_{k} \sigma_{k}\right|^{2}\right]^{1 / 2}=\left[\sum_{k}\left|\sum_{n>0} \hat{h}_{k}(n) \cos n x_{k}-b_{k} \sigma_{k}\right|^{2}\right]^{1 / 2}
$$


multiplication of the $k$ th increment by $\cos x_{k}$ and taking $\mathbf{E}_{k-1}$-expectation yields (by Proposition 5 and Lemma 6)

$$
\left\|\left[\sum_{k}\left|\frac{1}{2} \hat{h}_{k}(1)-\frac{2}{\pi} b_{k}\right|^{2}\right]^{1 / 2}\right\|_{1} \leqslant C\|h-f\|_{1}^{1 / 8}\|h\|_{1}^{7 / 8} .
$$

Since $b_{k}=\left(b_{k}\right)_{e}$, a convexity argument allows us to replace, in a previous inequality, $\hat{h}_{k}(1)$ by $\left(\hat{h}_{k}(1)\right)_{e}$. Combining with Lemma 7 , we conclude

$$
C^{-1}\|f\|_{1} \leqslant\left\|\left(\sum\left|b_{k}\right|^{2}\right)^{1 / 2}\right\|_{1} \leqslant C\|h-f\|_{1}^{1 / 16}\|h\|_{1}^{15 / 16}, \quad\|f\|_{1} \leqslant C\|h-f\|_{1},
$$

and thus Proposition 10.

\section{REFERENCES}

1. A. Bonami, Étude des coefficients de Fourier des fonctions de $L^{p}(G)$, Ann. Inst. Fourier (Grenoble) 20 (1970), 335-402.

2. J. Bourgain, On the embedding problem of $L^{1}$ in $L^{1} / H^{1}$, Bull. Soc. Math. Belg. 34 (1982), 187-194.

3. J. Bourgain and H. P. Rosenthal, Applications of the theory of semi-embeddings to Banach space theory, preprint.

4. J. Bourgain, New Banach space properties of the disc algebra and $H^{\infty}$, Acta Math. (to appear).

5. J. Garnett, Bounded analytic functions, Academic Press, New York, 1981.

6. J. Garnett and P. Jones, The distance in BMO to $L^{\infty}$, Ann. of Math. (2) 108 (1978), 373-393.

7. A. Garsia, Martingale inequalities, Math. Lecture Notes, Benjamin, New York, 1973.

8. J. Lindenstrauss and L. Tzafriri, Classical Banach spaces, Vol. I, Ergeb. Math. Grenzgeb., no. 92, Springer-Verlag, Berlin and New York, 1977.

9. A. Pekczysnki, Banach spaces of analytic functions and absolutely summing operators, CBMS Regional Conf. Ser. in Math., no. 30, Amer. Math. Soc., Providence, R.I., 1976.

10. E. Stein, Singular integrals and differentiability properties of functions, Princeton Univ. Press., Princeton, N.J., 1970.

11. Classes $H^{p}$, multiplicateurs et fonctions de Littlewood-Paley, C.R. Acad. Sci. Paris Sér. A 263 (1966), 716-719; 780-781.

Department of Mathematics, Vrije Universiteit Brussel, PleinlaAn 2-F7, 1050 Brussels, BELGIUM 